\title{
Huntington's disease-specific mis-splicing captured by human-mouse intersect-RNA-seq unveils pathogenic effectors and reduced splicing factors
}

Ainara Elorza ${ }^{1,2}$, Yamile Márquez ${ }^{3}$, Jorge R. Cabrera ${ }^{1,2}$, José Luis Sánchez-Trincado ${ }^{1,2}$, María Santos-Galindo ${ }^{1,2}$, Ivó H. Hernández ${ }^{1,2,4}$, Juan Ignacio Díaz-Hernández ${ }^{1,2}$, Ramón GarcíaEscudero ${ }^{5,6,7}$, Manuel Irimia ${ }^{3,8,9 *}$ and José J. Lucas ${ }^{1,2 *}$

${ }^{1}$ Center for Molecular Biology "Severo Ochoa” (CBMSO) CSIC/UAM, Madrid 28049, Spain

${ }^{2}$ Networking Research Center on Neurodegenerative Diseases (CIBERNED). Instituto de Salud Carlos III, Madrid 28031, Spain

${ }^{3}$ Centre for Genomic Regulation (CRG), Barcelona Institute for Science and Technology, 08003 Barcelona, Spain

${ }^{4}$ Departamento de Biología, Facultad de Ciencias, Universidad Autónoma de Madrid, Madrid 28049, Spain

${ }^{5}$ Molecular Oncology Unit, CIEMAT, Madrid 28040, Spain

${ }^{6}$ Biomedicine Research Institute, Hospital 12 Octubre, Madrid 28041, Spain

${ }^{7}$ Centro de Investigación Biomédica en Red de Cáncer (CIBERONC), Instituto de Salud Carlos III, Madrid 28029, Spain

${ }^{8}$ Universitat Pompeu Fabra, 08003, Barcelona, Spain

${ }^{9}$ ICREA, Barcelona, Spain.

*Corresponding authors: José J. Lucas

Center for Molecular Biology "Severo Ochoa” (CBMSO)

C/ Nicolás Cabrera,1. Campus UAM de Cantoblanco

28049 Madrid. Spain

Tel. +34 911964552

e-mail: jilucas@cbm.csic.es

Manuel Irimia

Centre for Genomic Regulation (CRG)

C/ Dr. Aiguader, 88

08003 Barcelona. Spain

Tel. +34933160212

e-mail: mirimia@gmail.com 


\section{Abstract}

Deregulated alternative splicing has been implicated in a wide range of pathologies. Deep RNAsequencing has revealed global mis-splicing signatures in multiple human diseases; however, for neurodegenerative diseases, these analyses are intrinsically hampered by neuronal loss and neuroinflammation in post-mortem brains. To infer splicing alterations relevant to Huntington's disease (HD) pathogenesis, here we performed intersect-RNA-seq analyses of human postmortem striatal tissue and of an early symptomatic mouse model in which neuronal loss and gliosis are not yet present. Together with a human/mouse parallel motif scan analysis, this approach allowed us to identify the shared mis-splicing signature triggered by the HD-causing mutation in both species and to infer upstream deregulated splicing factors. Moreover, we identified a plethora of downstream neurodegeneration-linked effector genes, whose aberrant splicing is associated with decreased protein levels in HD patients and mice. In summary, our intersect-RNA-seq approach unveiled the pathogenic contribution of mis-splicing to HD and could be readily applied to other neurodegenerative diseases for which bona fide animal models are available. 


\section{Introduction}

Alternative splicing (AS) of pre-mRNA is the differential processing of introns and exons to generate multiple transcript isoforms from individual genes, thereby increasing molecular diversity. However, when it is not properly executed, AS leads to mis-splicing, which may result in proteins with altered function and stability ${ }^{1}$. A number of splicing factors and other RNAbinding proteins (RBPs) are responsible for proper regulation of $\mathrm{AS}^{2}$, and growing evidence has implicated mis-splicing in a range of pathologies such as cancer ${ }^{3}$, muscular dystrophies ${ }^{4}$, autism $^{5,6}$ and neurodegenerative diseases ${ }^{7}$ such as Alzheimer's disease ${ }^{8,9}$, amyotrophic lateral sclerosis $^{10,11}$ and Huntington's disease ${ }^{12-15}$. Next-generation RNA sequencing (RNA-seq) has boosted investigation of global mis-splicing in diseased tissue. Nevertheless, while analysis of tumour or dystrophic muscle biopsies can be informative on early stage pathogenic mis-splicing, RNA-seq studies of neurodegenerative diseases using post-mortem brains are confounded by the dramatically altered cellular composition in end-state disease tissue due to neuronal loss and increased gliosis, the latter besides leading to a chronic inflammatory status. These caveats make it difficult to identify the causative molecular alterations that may serve as bases for therapeutic approaches.

Huntington's disease (HD) is a devastating neurological disorder characterized by prominent motor symptoms and marked atrophy of the nucleus striatum ${ }^{16}$. HD is caused by a polyglutamine (polyQ)-encoding CAG repeat expansion in the Huntingtin $(H T T)$ gene ${ }^{17}$. Similar pathogenic CAG mutations in different genes cause multiple dominant spinocerebellar ataxias (SCAs), including SCA-1, $-2,-3,-6,-7$ and $-17^{18}$ and there is evidence of toxicity being mediated by both the expanded CAG-containing mRNAs and the polyQ-containing proteins ${ }^{19,20}$. The proteins that interact with expanded CAG mRNA include splicing factors such as MBNL1 ${ }^{21}$, U2AF2 ${ }^{22}$ and SRSF6 $6^{15,23}$, the latter being also sequestered into the characteristic polyQ inclusion bodies found 
in HD brains ${ }^{13}$. All this has led to the proposal that splicing alterations may, at least in part, underlie $\mathrm{HD}^{12-15}$. In fact, hypothesis-driven studies have identified two mis-splicing events in the etiology of HD. Namely, a retained intron in the HTT gene giving rise to a highly toxic exon1encoded $\mathrm{N}$-terminal truncated form of mutant $\mathrm{Htt}{ }^{12}$, and elevated inclusion of tau (MAPT) exon 10 , leading to a pathogenic increase in tau isoforms with four tubulin binding repeats (4R-tau) ${ }^{13}$. However, analysis of global mis-splicing in striatum of HD patients is missing due to the limitations imposed by the altered cellular composition in post-mortem samples discussed above.

To investigate mis-splicing patterns potentially relevant to the onset of HD, here we performed at par RNA-seq analyses of striatum of HD patients and of a highly validated HD transgenic mouse model at an early disease stage, in which motor symptoms are due to circuit dysfunction prior to appearance of neuronal loss and gliosis. Intersection of both analyses yielded a shared mis-splicing signature triggered by the HD causing mutation in striatum of both species (Fig.1). Characterization of this signature allowed us to infer a network of upstream splicing factors deregulated in both species and to identify a plethora of downstream neurodegeneration-linked effector genes whose aberrant splicing led us to corroborate their decreased protein levels in HD striatum. 


\section{Results}

\section{Intersect-RNA-seq analysis of striatum of HD patients and early symptomatic mice identifies a} shared mis-splicing signature affecting movement disorder-related genes

Given the fully penetrant and dominant nature of the HD causing mutation, transgenic animal models that truly recapitulate symptoms and neuropathology have been generated. In particular, mice of the R6/1 model express a transgene driven by the endogenous Htt promoter that encodes the highly toxic exon1-encoded N-terminal form of $H t t$ with a CAG expansion resulting in a robust, yet slowly-progressing, motor phenotype ${ }^{24}$. At the age of 3.5 months, R6/1 mice in our colony already showed a clear motor coordination deficit $\left(p=5.0 \times 10^{-3}\right.$; Student's ttest; Supplementary Fig. 1a), but no significant striatal atrophy or neuropathology, as evidenced by volumetric analysis of the striatum together with stereological neuronal count and immunostaining with markers of gliosis (Supplementary Fig. 1b,c). Given the lack of gliosis and neuronal loss at this stage, we reasoned that a transcriptomic comparison of striatum from this mouse model and from human HD patients could reveal shared HD-specific pathogenic missplicing signatures triggered by the HD-mutation in both species, devoid of secondary alterationrelated artifacts. Therefore, we performed at par RNA-seq analyses of post-mortem striatum of HD patients (Vonsattel's grade 3-4) and matching control subjects $(n=3)$, and of striatum of 3.5 month-old R6/1 mice, together with matching controls $(n=3)$. Between 55 and 102 million reads (101-bp, pair-ended) were generated per sample (Fig. 2a). Differential gene expression analysis confirmed no alterations in the levels of neuronal and glial marker genes in the R6/1 RNA-seq samples (Fig. 2b). In contrast, as expected from the marked neuronal loss and gliosis of human HD post-mortem striatum ${ }^{25}$, we observed a strong decrease in the relative expression of neuronal marker genes $\left(p=8.0 \times 10^{-5}\right.$; Student's t-test) and an increase in glial-marker genes, particularly those of astrocytes ( $p=0.018$; Student's t-test) and microglia $\left(p=4.2 \times 10^{-3}\right.$; Student's t-test) (Fig. 2b). 
Next, we compared AS between HD and control samples in both species using three complementary software packages (vast-tools, rMATS and SANJUAN; see Methods for details). Combining the results obtained from each tool produced a list of 5,747 genes with at least one differentially spliced AS event in HD patients respect to control subjects (Fig. 2c, Supplementary Fig. 2 and Supplementary Table 1). Since isoform usage differs widely across cell types ${ }^{26,27}$ and is regulated upon inflammation ${ }^{28}$, a substantial fraction of the observed AS changes are expected to be due to the altered neuronal/glia cellular composition and/or to the pro-inflammatory signals in post-mortem HD tissue. In line with this, the number of differentially spliced genes in R6/1 mice respect to controls was much smaller (2,071 genes; Fig. 2c, Supplementary Fig. 2 and Supplementary Table 2). Remarkably, a total of 949 one-to-one orthologs were differentially spliced in both human and mouse $\left(p=6.12 \times 10^{-3}\right.$, two-sided Fisher's Exact test; Fig. $2 c$ and Supplementary Tables 3-10). This set of genes are expected to reflect AS alterations that are mainly due to the toxicity of the HD mutation independently of altered cellular contentassociated artifacts in the human samples. Accordingly, this shared mis-splicing signature represents an important fraction of all mis-spliced genes in the mouse model (45.8\%) but only a minority of those observed in human tissue (16.5\%). Interestingly, Gene Ontology analysis revealed that common mis-spliced genes were strongly enriched for functions related to basal ganglia and movement disorders (including HD)(Fig. 2d and Supplementary Table 11), suggesting that these shared AS alterations in striatum are a plausible contributor to HD pathogenesis.

\section{Mis-splicing of neurodegeneration-linked genes correlates with reduced protein levels}

To further explore the pathogenic relevance of mis-spliced genes, we next focused on a subset of manually curated genes with differential AS in both species and whose mutations cause monogenic forms of neurodegeneration in humans: CCDC88C (Coiled-coil domain containing 88C linked to SCA40 -spinocerebellar ataxia 40-, OMIM \#616053), KCTD17 (Potassium channel tetramerization domain containing 17 linked to DYT26 -myoclonic dystonia-26-, OMIM 
\#616398), SYNJ1 (Synaptojanin 1 linked to PARK20 -early onset Parkinson disease-20-, OMIM \#615530), VPS13C (Vacuolar protein sorting 13 homolog C linked to PARK23 -early onset Parkinson disease-23-, OMIM \#616840), TRPM7 (Transient receptor potential cation channel subfamily M member 7 linked to ALSP/DC -amyotrophic lateral sclerosis-parkinsonism/dementia complex-, OMIM \#105500) and SLC9A5 (Solute carrier family 9 member A5 also known as $\mathrm{Na}(+) / \mathrm{H}(+)$ exchanger 5 that has been suggested to be linked to EKD2 -episodic kinesigenic dyskinesia 2-, as it maps to the $16 q 13-q 22.1$ candidate region $^{29}$ ) (Fig. 3a). Using $\operatorname{VastDB}^{30}$, we identified the orthologous AS events that were differentially spliced in both species, and which corresponded to skipped exons (SE) in all cases except for TRPM7, which had a differentially retained intron (RI) (Fig. 3b). All these mis-spliced events detected by RNA-seq were validated by RT-PCR assays using striatal mRNA from an independent set of $R 6 / 1$ and wild-type mice $(n=5$;

Fig. 3c).

Next, we tested whether the splicing alterations observed in striatum of R6/1 mice in these neurodegeneration-associated genes correlated with changes in their protein levels. Western blot analyses revealed a significant decrease in protein levels for all of them except for Vps13c, which also showed a trend of reduced protein levels respect to the control samples (Fig. 4). Since both recessive mutations and decreased protein levels may result in decreased protein function, these data suggest that the observed global mis-splicing in striatum of HD patients and mice may have a pathogenic effect by diminishing protein levels of multiple neurodegenerationlinked genes.

\section{Parallel RBP motif enrichment analysis reveals splicing factors altered in HD striatum}

We next looked for potential RBPs that may be responsible for the shared mis-splicing HD signature. For this purpose, we searched for known binding motifs for RBPs ${ }^{31}$ inside and around orthologous exons that were mis-regulated in both species. We reasoned that motifs associated 
with relevant RBPs would be similarly enriched in both exons sets. Since vast-tools directly provides exon-based homology information between the two species, we focused on 73 orthologous alternative exons detected by this software as mis-spliced in both human and mouse ( $|\triangle \mathrm{PSI}| \geq 15)$ (Supplementary Table 12). As a control gene set, we used 914 alternatively spliced exons whose usage was not altered in $\mathrm{HD}$ and $\mathrm{R} 6 / 1$ samples respect to controls (|DPSI| <5) (Fig. 5a). Motifs associated with six RBP families showed significant enrichments in the equivalent positions in both species: binding motifs of TIA1, U2AF2, HNRNPC and PTBP were enriched in the upstream intronic sequences and of RBFOX and ELAVL in downstream intronic sequences (Fig. 5b).

Western blot analysis of these RBPs in striatum of R6/1 and control mice revealed a significant decrease in protein levels for nine different members belonging to five of the six identified RBP families. Specifically, we found decreased levels for TIA1 (71\%, $p=9.2 \times 10^{-4}$; Student's t-test), U2AF2 (26\%, $p=2.2 \times 10^{-3}$; Student's t-test), RBFOX1 ( $28 \% p=0.016$; Student's t-test), RBFOX2 (46\%, $p=8.5 \times 10^{-3}$; Student's t-test), RBFOX3 $\left(60 \%, p=1.3 \times 10^{-5}\right.$; Student's t-test), ELAVL4 (76\%, $p=1.5 \times 10^{-4} ;$ Student's t-test), ELAVL2 (55\%, $p=2.3 \times 10^{-5}$; Student's t-test), ELAVL1 (30\%, $p=2.9 \times 10^{-}$ 4; Student's t-test) and HNRNPC (66\%, $p=1.9 \times 10^{-3}$; Student's t-test) (Fig. 6). In contrast, the protein levels of ELAVL3 did not show any change in R6/1 striatum, neither did the two paralogs of the PTBP family, which were preferentially expressed in astrocytes ${ }^{32}$ (Supplementary Fig. 3).

\section{Validation of altered splicing factors and pathogenic downstream effectors in striatum of HD patients}

Finally, we assessed whether the protein-level alterations observed in R6/1 striatum for both splicing factors and neurodegenerative-associated genes reflected matching alterations in the striatum of HD patients, as expected from our intersect-RNA-seq analysis. Regarding the splicing factors, we found decreased protein levels for TIA1 (91\%, $p=0.036$; Student's t-test), U2AF2 (65\%, $p=0.045$; Student's t-test), RBFOX1 (76\%, $p=5.3 \times 10^{-4}$; Student's t-test), RBFOX2 (46\%, 
$p=4.6 \times 10^{-3}$; Student's t-test), RBFOX3 $\left(96 \%, p=3.3 \times 10^{-3}\right.$; Student's t-test) and ELAVL2 $(62 \%$, $p=1.8 \times 10^{-4} ;$ Student's t-test). Besides, a similar trend for decreased protein levels $(53 \%, p=0.17$; Student's t-test) was also observed for HNRNPC (Fig. 7a). For the subset of neurodegenerationlinked mis-spliced genes, we observed a sharp decrease in protein levels for virtually all of them (Fig. 7b). More precisely, for CCDC88C we found a 90\% decrease in a low molecular weight form $\left(p=3.2 \times 10^{-5}\right.$; Student's t-test) and a trend for decrease in the canonical $228 \mathrm{kDa}$ form $(60 \%$, $p=0.081$; Student's t-test), for KCTD17 we observed a 58\% decrease $\left(p=1.7 \times 10^{-3}\right.$; Student's $t$ test) in the monomeric ( $36 \mathrm{kDa}$ ) form and a $59 \%$ decrease ( $p=0.048$; Student's t-test) in the pentameric form (data not shown), and we also observed decreased levels of SYNJ1 (56\%, $p=0.021 ;$ Student's t-test), TRPM7 (82\%, $p=6.0 \times 10^{-3} ;$ Student's t-test), VPS13C $(69 \%, p=0.012$; Student's t-test) and SLC9A5 ( $83 \%, p=1.0 \times 10^{-3}$; Student's t-test) (Fig. 7b). Altogether, these results indicate that the mis-splicing HD signature and the candidate splicing factors identified by our intersect-RNA-seq and parallel motif scan analyses show high validation rates also at the protein level in striatum of HD patients. 


\section{Discussion}

Here we report a novel approach to identify potentially pathological signatures of mis-splicing in HD patients overcoming the artifacts associated with altered cell type composition and neuroinflammation in human post-mortem brain tissues. We performed at par RNA-seq analysis of striatal tissue from a mouse model of HD at an early symptomatic stage and from human post-mortem HD brain, whose intersection revealed a shared HD-specific mis-splicing signature affecting 949 one-to-one orthologous genes. These included a subset of genes that had been previously involved in neurodegenerative movement disorders and that we showed to have reduced protein levels in the mouse model as well as in HD patients. In addition, by humanmouse parallel complementary motif searches on common mis-spliced events, we inferred a network of candidate upstream splicing factors with reduced protein levels in both species.

A previous report also addressed global mis-splicing in human post-mortem HD tissue by RNAseq analysis ${ }^{14}$. However, instead of studying the striatum, which is the brain region primarily and most affected in the disease, this study focused on BA4 motor cortex, which shows a less marked atrophy in post-mortem tissue. Nevertheless, it is well documented that the cortex also degenerates early in the course of disease ${ }^{33}$, and therefore is likely affected by similar caveats associated to neurodegeneration and neuroinflammation. In line with this, Lin and co-workers detected enrichment of 15 splicing factor motifs in their set of differential AS events, among which only PTBP1 overlaps with the splicing factors detected in our analysis. However, PTBP1 is preferentially expressed in astrocytes ${ }^{32}$ and is not altered at the protein level neither in human or mouse HD striatum, unlike the other five families of splicing factors that we detected through our parallel motif scan. Besides, out of the nine candidate splicing factors that show altered mRNA levels in Lin's work, the majority also change in human striatum according to our RNAseq data, but only one is altered in striatum of early symptomatic mice. Overall, these 
observations point to neurodegeneration-associated confounding factors and strengthen the notion that, by refining mis-splicing analysis by intersecting results from human patients and an early mouse model, neurodegeneration- and neuroinflammation-associated artifacts are cleared out in our study.

Eight additional neurological disorders including the spinal and bulbar muscular atrophy (SBMA) and several SCAs are caused by expanded CAG repeats in coding sequences of different genes that give rise to expanded polyQ containing proteins ${ }^{18}$. Given the similarity in the causing mutations, the molecular mechanisms in HD pathogenesis -including splicing alterations-, are likely contributors to the other CAG repeat polyglutaminopathies. Most of these diseases show maximal affectation in cerebellum and the analysis of global mis-splicing in post-mortem cerebellar patient tissue for these diseases poses the same challenges as those addressed here for HD striatum. Since there are also good animal models for these triplet repeat disorders ${ }^{34}$, at par early mouse model RNA-seq to identify the intersecting mis-splicing signature and parallel motif scans to infer associated splicing regulators will be useful to determine whether similar AS-related pathogenic mechanisms underlie the different CAG trinucleotide repeat disorders. Similarly, the human-mouse intersect-RNA-seq approach might be applied to any neurodegenerative disease for which animal models with construct validity are available and for which alterations in RNA processing are suspected as a pathogenic mechanism. These include the amyotrophic lateral sclerosis/frontotemporal dementia disease continuum (ALS/FTD) ${ }^{35}$, particularly the forms associated to mutations in genes encoding RBPs (TDP43 and FUS) or affecting formation of RNA foci (C9orf72) ${ }^{36}$ for which bona fide animal models exist ${ }^{1}$.

Pathological expanded CAG-containing mRNAs adopt a hairpin conformation ${ }^{37}$ and some of the altered splicing factors detected here are likely to be affected by direct and aberrant interaction with expanded CAG mRNA. This is in fact the case of U2AF2 $2^{15,22}$ and HNRNPC ${ }^{15}$. Both U2AF2 and 
HNRNPC bind to U-rich motifs, as also do TIA1 and the ELAVL family, all identified here as misregulated in human HD patients and the mouse model. Since they compete for similar target mRNAs and may be regulated by common interactors, it is conceivable that deregulation of TIA1 and ELAVL might be secondary to U2AF2 and HNRNPC alterations driven by their interaction with expanded CAG repeats. The RBFOX family is the only one altered in our study that does not bind U-rich motifs, as it binds the GCAUG consensus ${ }^{38}$. However, RBFOX splicing factors also act through a multiprotein complex called LASR (Large Assembly of Splicing Regulators) that includes HNRNPC ${ }^{39}$ and it is conceivable that sequestration of HNRNPC by expanded CAG-HTT exon1 RNA ${ }^{15}$ might subsequently affect RBFOX binding and stability.

In summary, by intersect-RNA-seq analysis of at par-processed early mouse model and human post-mortem brain tissue, we have identified a HD-specific mis-splicing signature that allowed us to infer altered upstream splicing factors and downstream pathogenic effectors that can become new therapeutic targets for HD. Moreover, this approach can be applied to investigate the potential relevance of altered AS in other neurodegenerative diseases. 


\section{Acknowledgements}

This work was supported by CIBERNED-ISCIII collaborative grants PI2015-2/06-3 and PI2018/06-

1; by grants: SAF2015-65371-R (MINECO/AEI/FEDER, UE); RTI2018-096322-B-I00

(MCIU/AEI/FEDER, UE) from Spanish Ministry of Economy and Competitiveness/Ministry of Science, Innovation and Universities (MINECO/MICINN) to JJL and BFU2017-89201-P (MINECO/AEI/FEDER, UE) to MI; PI18/00263 from the Instituto de Salud Carlos III (Ministry of Economy, Industry and Competitiveness) co-funded by the European Regional Development Fund- to RG-E; institutional grant from Fundación Ramón Areces to CBMSO ; Fundación BBVA to JJL; and by the European Research Council under the European Union's Horizon 2020 research and innovation program ERC-StG-LS2-637591 to MI. Human tissue was obtained from Institute of Neuropathology (HUB-ICO-IDIBELL) Brain Bank, the Neurological Tissue Bank of the IDIBAPS Biobank, the Banco de Tejidos Fundación CIEN, and the Netherlands Brain Bank. We thank Juan Valcárcel for scientific advice. We also thank excellent technical assistance by Miriam Lucas and by the following core facilities: CBMSO-Genomics \& Massive Sequencing, CBMSO-Animal Facility and the CETA-CIEMAT computing center. We also thank Alvaro Lucas for help with illustrations. 


\section{Materials and Methods}

\section{Human brain tissue samples}

Brain specimens used in this study from striatum of HD patients and controls (CTRL) were provided by Institute of Neuropathology Brain Bank (HUB-ICO-IDIBELL, Hospitalet de Llobregat, Spain), the Neurological Tissue Bank of the IDIBAPS Biobank (Barcelona, Spain), the Banco de Tejidos Fundación Cien (BT-CIEN, Madrid, Spain) and the Netherlands Brain Bank (Amsterdam, The Netherlands). Written informed consent for brain removal after death for diagnostic and research purposes was obtained from brain donors and/or next of kin. Procedures, information and consent forms have been approved by the Bioethics Subcommittee of Consejo Superior de Investigaciones Científicas (CSIC, Madrid, Spain). The post-mortem interval in tissue processing was between 5:00 and 7:45 $\mathrm{h}$ for RNA-seq analysis and between 05:00 and 23:30 $\mathrm{h}$ for Western blot analyses. The neuropathological examination in HD cases revealed a diagnosis of $\mathrm{HD}$ grade 3-4 following Vonsattel's criteria ${ }^{25}$.

\section{Mice}

R6/1 transgenic mice for the human exon-1-Htt gene ${ }^{24}$ in B6CBAF1 background were bred and housed at the Centro de Biología Molecular Severo Ochoa animal facility. Mice were housed four per cage with food and water available ad libitum and maintained in a temperature-controlled environment on a 12/12 h light-dark cycle with light onset at 08:00. Animal housing and maintenance protocols followed the guidelines of Council of Europe Convention ETS123. Animal experiments were performed under protocols (PROEX293/15) approved by the Centro de Biología Molecular Severo Ochoa Institutional Animal Care and Utilization Committee (Comité de Ética de Experimentación Animal del CBM, CEEA-CBM), Madrid, Spain.

\section{Rotarod test}


Motor coordination was assessed with an accelerating rotarod apparatus (Ugo Basile, Comerio, Italy). Mice were trained during two consecutive days, the first day: 4 trials at fixed $4 \mathrm{rpm}$ for 1 min each, the second day: 4 trials for 2 min (the first minute at $4 \mathrm{rpm}$ and the second minute at $8 \mathrm{rpm}$ ). On the third day rotarod was set to accelerate from 4 to $40 \mathrm{rpm}$ over $5 \mathrm{~min}$ and mice were tested in 4 trials. The latency to fall from the rotarod was measured as a mean of the 4 accelerating trials.

\section{Tissue preparation for staining}

WT and R6/1 mice euthanasia was performed using $\mathrm{CO} 2$. Brain was quickly extracted and the left hemisphere was immersed in $4 \%$ paraformaldehyde overnight. After profuse washing in PBS, hemispheres were immersed in sucrose $30 \%$ in PBS for at least $72 \mathrm{~h}$ and then included in OCT (Optimum Cutting Temperature compound, Tissue-Tek, Sakura Finetek Europe, ref. 4583), frozen and stored at $-80^{\circ} \mathrm{C}$ until use. Mouse sagittal and coronal sections ( $30 \mu \mathrm{m}$ thick) were sequentially cut on a cryostat (Thermo Scientific), collected and stored free floating in glycolcontaining solution ( $30 \%$ glycerol, $30 \%$ ethylene glycol in $0.02 \mathrm{M}$ phosphate buffer) at $-20^{\circ} \mathrm{C}$.

\section{Immunohistochemistry}

Sagittal sections were first washed in PBS and then immersed in $0.3 \% \mathrm{H} 2 \mathrm{O} 2$ in PBS for 45 min to quench endogenous peroxidase activity. After PBS-washes, sections were immersed for $1 \mathrm{~h}$ in blocking solution (PBS containing $0.5 \%$ FBS, $0.3 \%$ Triton X-100 and 1\% BSA) and incubated overnight at $4{ }^{\circ} \mathrm{C}$ with anti-DARPP32 (1:5000, Chemicon, AB1656) or anti-IBA1 (1:500, Wako, 019-19741) diluted in blocking solution. After washing, brain sections were incubated first with biotinylated goat anti-rabbit secondary antibody and then with avidin-biotin complex using the Elite Vectastain kit (Vector Laboratories, PK-6101). Chromogen reactions were performed with diaminobenzidine (SIGMAFAST DAB, Sigma, D4293) for $10 \mathrm{~min}$. Mouse sections were mounted 
on glass slides and coverslipped with Mowiol (Calbiochem, Cat. 475904). Images were captured using an Olympus BX41 microscope with an Olympus camera DP-70 (Olympus Denmark A/S).

\section{Striatal volumetry}

Coronal sections ( $30 \mu \mathrm{m}$ thick) were cut on a cryostat and every sixth section was counterstained with toluidine blue pH 4.0 (1 g/l Toluidine Blue, Sigma, 198161 in $0.8 \mathrm{M}$ glacial acetic acid). Digital images were captured at a 2.5x magnification (Canon EOS 450D digital camera) and striatal areas from 19-22 sections for each animal were calculated by means of the ImageJ software ${ }^{40}$. Considering a separation of $180 \mu \mathrm{m}$ between each section, total structure volume in each mouse was calculated.

\section{Stereology}

Coronal sections (30- $\mu \mathrm{m}$ thick) counterstained with toluidine blue $\mathrm{pH} 4.0(1 \mathrm{~g} / \mathrm{l}$ toluidine blue (Sigma, 198161), $0.8 \mathrm{M}$ glacial acetic acid) from the volumetric analysis were used. Sections containing striatum were selected and the 15 most central sections were analyzed. One randomly selected $60 \mu \mathrm{m} \times 60 \mu \mathrm{m}$ optical dissector at $60 \times$ magnification with an Olympus BX41 microscope with an Olympus camera DP-70 (Olympus Denmark A/S) was analyzed in each section. Total neuronal cell number per dissector was assessed by a researcher blind to genotype. Striatal neuronal cell density was calculated and compared for WT $(n=3)$ and R6/1 mice $(n=3)$.

\section{RNA sequencing}

Total RNA was isolated using the Maxwell 16 LEV simplyRNA Tissue Kit (Promega, AS1280) and quantified by Qubit ${ }^{\circledR}$ RNA BR Assay kit (Thermo Fisher Scientific) and the RNA integrity number (RIN) was estimated by using RNA 6000 Nano Bioanalyzer 2100 Assay (Agilent). The RNA-seq libraries were prepared with KAPA Stranded mRNA-Seq Illumina ${ }^{\circledR}$ Platforms Kit (Roche) 
following the manufacturer's recommendations. Briefly, $500 \mathrm{ng}$ of total RNA was used as the input material, the poly-A fraction was enriched with oligo-dT magnetic beads and the mRNA was fragmented. The strand specificity was achieved during the second strand synthesis performed in the presence of dUTP instead of dTTP. The blunt-ended double stranded cDNA was 3'adenylated and Illumina platform compatible adaptors with unique dual indexes and unique molecular identifiers (Integrated DNA Technologies) were ligated. The ligation product was enriched with 15 PCR cycles and the final library was validated on an Agilent 2100 Bioanalyzer with the DNA 7500 assay. The libraries were sequenced on HiSeq 2500 (Illumina, Inc) with a read length of $2 \times 101 \mathrm{bp}$ using HiSeq 4000 SBS kit in a fraction of a HiSeq 4000 PE Cluster kit sequencing flow cell lane generating 55-102 million paired-end reads per sample. Image analysis, base calling and quality scoring of the run were processed using the manufacturer's software Real Time Analysis (RTA 2.7.7).

\section{RNA-seq analysis}

Differentially expressed genes between the biological groups were analyzed using vast-tools v1.1.0 compare_expr with default parameters ${ }^{30}$. To verify correct clustering according to genotype, hierarchical clustering of cRPKM expression values was performed using "hclust" and "heatmap.2" R packages over significantly changed genes in HD vs CTRL and R6/1 vs WT mice (Benjamini-Hochberg correction method). Cell-type-marker genes (neurons, astrocytes, oligodendrocytes and microglia) as described previously ${ }^{14}$ were analyzed by calculating their geometric mean of cRPKM values for each cell type excluding those genes with cRPKM null value in at least one sample and data were plotted and analyzed using GraphPad software (La Jolla, CA).

To identify differential alternative splicing events between the two sample groups per species we used three complementary software: (1) vast-tools v1.1.0, using human (hg19; vastdb.hsa.13.11.15) or mouse ( $\mathrm{mm9}$; vastdb.mmu.13.11.15) junction libraries for the align and 
combine modules ${ }^{30}$. Differential splicing analysis was done using the module compare with default parameters $(|\Delta \mathrm{PS}| \mid \geq 15$ and a minimum $\triangle \mathrm{PSI}$ of 5 in the same direction among all replicates). (2) rMATS, v3.0.8 $8^{41}$, utilizing TopHat2 ${ }^{30}$ to align fastq reads against the GRCh38.p2 (dec. 2014) and GRCm38.p3 (jan. 2012) genomes together with a custom splice-junction library. For differential splicing analyses, default parameters were used (FDR $<5 \%$ ). (3) SANJUAN v1.0beta, which detects de-novo splicing junctions and AS events (https://github.com/ppapasaikas/SANJUAN). For differential splicing analyses, medium confidence level of constraints on differential splicing junctions and $|\triangle P S I| \geq 15$ were used. The relative contribution of each tool to the detection of mis-spliced genes is shown in Supplementary Fig. 2 and Supplementary Tables 3-8.

The entire RNA-seq dataset will be available at the Gene Expression Omnibus (GEO) database with the accession number XXXXX.

\section{Ingenuity Pathway Analysis (IPA) analysis}

Genes mis-spliced in both human and mouse were annotated to diseases and functions using Ingenuity Pathway Analysis (IPA) software (http://www.ingenuity.com). The analysis was performed in October 2019 and the results were filtered for neurological diseases related functions.

\section{Semiquantitative real-time PCR validation}

Selected events were evaluated with semiquantitative RT-PCR. Total RNA (500 ng) was reversetranscribed using Invitrogen Superscript IV reverse transcriptase and cDNA (50 ng) was amplified with gene/exon-specific primers. PCR products were resolved on $2 \%$ high-resolution Metaphor agarose gels (Lonza).

\section{Primers}




\begin{tabular}{|c|c|c|}
\hline \multirow[t]{2}{*}{ Ccdc88c } & Forward & 5'-GGATTGGAGCCAAAGCCCTAG-3' \\
\hline & Reverse & 5'-CTGAGGTTGAAAAGGTCCGGG-3' \\
\hline \multirow[t]{2}{*}{ Kctd17 } & Forward & 5'-GGTGAACATCGGCTCCTCCTA-3' \\
\hline & Reverse & 5'-GGGTGCCTCTGGCTTGTAATAG-3' \\
\hline \multirow[t]{2}{*}{ Synj1 } & Forward & 5'-GCTGAGGGTGAAGAGGAGTGA-3' \\
\hline & Reverse & 5'-GCTGATGGCATCTCGGATGTT-3' \\
\hline \multirow[t]{2}{*}{ Vps13c } & Forward & 5'-CCCAGACTCTAGAGCCCAAGA-3' \\
\hline & Reverse & 5'-GCTTGAGGGGAAGGCTGATTAC-3' \\
\hline \multirow[t]{2}{*}{ Trpm7 } & Forward & 5'-TGCCGAATTGAAGAAGCCCTT-3' \\
\hline & Reverse & 5'-TGTGAATGTCTGTAATTTTCACTTGA-3' \\
\hline \multirow[t]{2}{*}{ Slc9a5 } & Forward & 5'-TCCAGCTTCTGTGACGTATGC-3' \\
\hline & Reverse & 5'-AGTATTGCTTTTCTGGGAACACC-3' \\
\hline
\end{tabular}

\section{Western Blot}

Samples from human brain were stored at $-80^{\circ} \mathrm{C}$ and ground with a mortar in a frozen environment with liquid nitrogen to prevent thawing of the samples, resulting in tissue powder. Mouse brains were quickly dissected on an ice-cold plate and the different structures stored at $-80^{\circ} \mathrm{C}$. Human and mouse protein extracts were prepared by homogenizing brain structures in ice-cold extraction buffer (20 mM HEPES pH 7.4, 100 mM NaCl, 20 mM NaF, 1\% Triton X-100, 1 $\mathrm{mM}$ sodium orthovanadate, $1 \mu \mathrm{M}$ okadaic acid, $5 \mathrm{mM}$ sodium pyrophosphate, $30 \mathrm{mM} \beta$ glycerophosphate, 5 mM EDTA, protease inhibitors (Complete, Roche, Cat. No 11697498001)). Homogenates were centrifuged at $15000 \mathrm{rpm}$ for $15 \mathrm{~min}$ at $4^{\circ} \mathrm{C}$. The resulting supernatant was collected, and protein content determined by Quick Start Bradford Protein Assay (Bio-Rad, 5000203). $20 \mu \mathrm{g}$ of total protein were electrophoresed on $10 \%$ SDS-polyacrylamide gel, transferred to a nitrocellulose blotting membrane (Amersham Protran $0.45 \mu \mathrm{m}$, GE Healthcare Life Sciences, 10600002) and blocked in TBS-T (150 mM NaCl, $20 \mathrm{mM}$ Tris-HCl, $\mathrm{pH} 7.5,0.1 \%$ Tween 20) supplemented with $5 \%$ non-fat dry milk. Membranes were incubated overnight at $4^{\circ} \mathrm{C}$ with the primary antibody in TBS-T supplemented with $5 \%$ non-fat dry milk, washed with TBS-T and next 
incubated with HRP-conjugated anti-mouse IgG (1:2000, DAKO, P0447) or anti-rabbit IgG (1:2000, DAKO, P0448) and developed using the ECL detection kit (PerkinElmer, NEL105001EA).

\section{Antibodies}

Rabbit anti-CCDC88C (1:500, Sigma, HPA005832), rabbit anti-KCTD17 (1:500, Thermo Fisher, PA5-72101), mouse anti-SYNJ1 (1:500, Thermo Fisher, MA3-936), rabbit anti-TRPM7 (1:1000, Novus Biologicals, NBP2-20739), rabbit anti-SLC9A5 (1:500, Abcam, ab191528), rabbit anti-TIA1 (1:1000, Abcam, ab40693), rabbit anti-U2AF2 (1:500, Santa Cruz, sc-53942), mouse anti-RBFOX1 (1:2000 for mouse and 1:1000 for human samples, Merk Millipore, MABE985), mouse antiRBFOX2 (1:500, Abcam, ab57154), rabbit anti-RBFOX3 (1:1000, Merk Millipore, MAB377), rabbit anti-ELAVL4 (1:500, Abcam, ab96474), rabbit anti-ELAVL2 (1:500, Abcam, ab96471), rabbit antiELAVL1 (1:500, Abcam, ab200342), rabbit anti-HNRNPC (1:500, Abcam, ab10294), rabbit antiELAVL3 (1:500, Abcam, ab129254), rabbit anti-PTBP1 (1:1000, Abcam, ab5642), rabbit antiPTBP2 (1:2000, Merk Millipore, ABE431), mouse anti- $\beta$-ACTIN (1:25000, Sigma, A2228), rabbit anti-VINCULIN(1:20000, DAKO, P0448).

\section{Motif enrichment analysis}

A total of 73 orthologous exons with differential splicing in both human and mouse were evaluated in parallel for RBPs motif enrichment. As a control set, we selected 914 conserved AS exons with no detectable changes $(|\Delta \mathrm{PSI}|<5)$ in the RNA-seq experiments of both species. RBP motifs deposited in the CIS-BP database (http://cisbp-rna.ccbr.utoronto.ca/index.php; downloaded july 2016) were scanned separately in exons, 250 intronic bases upstream the exon (3' splicing signal - 3'ss, excluding 20bp within the $3^{\prime}$ splice site) and 250 intronic bases downstream the exon (5'splicing signal -5 'ss, excluding $6 \mathrm{bp}$ within the $5^{\prime}$ splice site). Motif occurrences (log-odd $\geq 6$ for the respective RBP Position Weight Matrix) for each RBP were counted for regulated and control exons respectively. A motif can be counted zero or once in a 
given sequence. Motif enrichment for each RBP in regulated AS exons compared to the control set was evaluated using Fisher's exact test. To correct for multiple testing, Benjamini-Hochberg FDR was used for adjustment of $p$-values. Enrichment was considered significant when the adjusted $\mathrm{P}<0.01$.

\section{Data analysis}

Statistical analysis was performed with SPSS 21.0 (SPSS ${ }^{\circledR}$ Statistic IBM ${ }^{\circledR}$ ). Data are represented as Mean \pm SEM (Standard Error of the Mean). The normality of the data was analyzed by ShapiroWilk. For two-group comparison, two-tailed Student's t-test was performed (data with normal distribution) or Mann-Whitney U-test (data with non-normal distribution) was performed. A critical value for significance of $P<0.05$ was used throughout the study. Benjamini-Hochberg correction was applied for multiple testing in RNA-seq analysis and motif enrichment analysis, were enrichment was considered significant when the adjusted $P<0.01$. 


\section{References}

1. Montes, M., Sanford, B.L., Comiskey, D.F. \& Chandler, D.S. RNA Splicing and Disease: Animal Models to Therapies. Trends Genet 35, 68-87 (2019).

2. Ule, J. \& Blencowe, B.J. Alternative Splicing Regulatory Networks: Functions, Mechanisms, and Evolution. Molecular cell 76, 329-345 (2019).

3. Srebrow, A. \& Kornblihtt, A.R. The connection between splicing and cancer. Journal of cell science 119, 2635-2641 (2006).

4. Scotti, M.M. \& Swanson, M.S. RNA mis-splicing in disease. Nat Rev Genet 17, 19-32 (2016).

5. Irimia, M., et al. A highly conserved program of neuronal microexons is misregulated in autistic brains. Cell 159, 1511-1523 (2014).

6. Parras, A., et al. Autism-like phenotype and risk gene mRNA deadenylation by CPEB4 mis-splicing. Nature 560, 441-446 (2018).

7. Nik, S. \& Bowman, T.V. Splicing and neurodegeneration: Insights and mechanisms. Wiley Interdiscip Rev RNA 10, e1532 (2019).

8. Raj, T., et al. Integrative transcriptome analyses of the aging brain implicate altered splicing in Alzheimer's disease susceptibility. Nature genetics 50, 1584-1592 (2018).

9. Hsieh, Y.C., et al. Tau-Mediated Disruption of the Spliceosome Triggers Cryptic RNA Splicing and Neurodegeneration in Alzheimer's Disease. Cell reports 29, 301-316 e310 (2019).

10. Luisier, R., et al. Intron retention and nuclear loss of SFPQ are molecular hallmarks of ALS. Nature communications 9, 2010 (2018).

11. Fratta, P., et al. Mice with endogenous TDP-43 mutations exhibit gain of splicing function and characteristics of amyotrophic lateral sclerosis. The EMBO journal 37(2018).

12. Sathasivam, K., et al. Aberrant splicing of HTT generates the pathogenic exon 1 protein in Huntington disease. Proceedings of the National Academy of Sciences of the United States of America 110, 2366-2370 (2013).

13. Fernandez-Nogales, M., et al. Huntington's disease is a four-repeat tauopathy with tau nuclear rods. Nature medicine 20, 881-885 (2014).

14. Lin, L., et al. Transcriptome sequencing reveals aberrant alternative splicing in Huntington's disease. Human molecular genetics 25, 3454-3466 (2016).

15. Schilling, J., et al. Deregulated Splicing Is a Major Mechanism of RNA-Induced Toxicity in Huntington's Disease. J Mol Biol 431, 1869-1877 (2019).

16. Walker, F.O. Huntington's disease. Lancet (London, England) 369, 218-228 (2007).

17. Huntington's Disease Collaborative Research Group. A novel gene containing a trinucleotide repeat that is expanded and unstable on Huntington's disease chromosomes. Cell 72, 971-983 (1993).

18. Orr, H.T. \& Zoghbi, H.Y. Trinucleotide repeat disorders. Annual review of neuroscience 30, 575-621 (2007).

19. Li, L.B., Yu, Z., Teng, X. \& Bonini, N.M. RNA toxicity is a component of ataxin-3 degeneration in Drosophila. Nature 453, 1107-1111 (2008).

20. Shieh, S.Y. \& Bonini, N.M. Genes and pathways affected by CAG-repeat RNA-based toxicity in Drosophila. Human molecular genetics 20, 4810-4821 (2011).

21. Mykowska, A., Sobczak, K., Wojciechowska, M., Kozlowski, P. \& Krzyzosiak, W.J. CAG repeats mimic CUG repeats in the misregulation of alternative splicing. Nucleic acids research 39, 8938-8951 (2011).

22. Tsoi, H., Lau, C.K., Lau, K.F. \& Chan, H.Y. Perturbation of U2AF65/NXF1-mediated RNA nuclear export enhances RNA toxicity in polyQ diseases. Human molecular genetics 20, 3787-3797 (2011). 
23. Neueder, A., Dumas, A.A., Benjamin, A.C. \& Bates, G.P. Regulatory mechanisms of incomplete huntingtin mRNA splicing. Nature communications 9, 3955 (2018).

24. Mangiarini, L., et al. Exon 1 of the HD gene with an expanded CAG repeat is sufficient to cause a progressive neurological phenotype in transgenic mice. Cell 87, 493-506 (1996).

25. Vonsattel, J.P., et al. Neuropathological classification of Huntington's disease. Journal of neuropathology and experimental neurology 44, 559-577 (1985).

26. Ellis, J.D., et al. Tissue-specific alternative splicing remodels protein-protein interaction networks. Molecular cell 46, 884-892 (2012).

27. Heinzen, E.L., et al. Tissue-specific genetic control of splicing: implications for the study of complex traits. PLoS Biol 6, e1 (2008).

28. Ortis, F., et al. Cytokines interleukin-1beta and tumor necrosis factor-alpha regulate different transcriptional and alternative splicing networks in primary beta-cells. Diabetes 59, 358-374 (2010).

29. Spacey, S.D., et al. Mutation analysis of the sodium/hydrogen exchanger gene (NHE5) in familial paroxysmal kinesigenic dyskinesia. J Neural Transm (Vienna) 109, 1189-1194 (2002).

30. Tapial, J., et al. An atlas of alternative splicing profiles and functional associations reveals new regulatory programs and genes that simultaneously express multiple major isoforms. Genome research 27, 1759-1768 (2017).

31. Ray, D., et al. Rapid and systematic analysis of the RNA recognition specificities of RNAbinding proteins. Nat Biotechnol 27, 667-670 (2009).

32. https://www.proteinatlas.org/ENSG00000011304-PTBP1/tissue/cerebral+cortex.

33. Rosas, H.D., et al. Regional and progressive thinning of the cortical ribbon in Huntington's disease. Neurology 58, 695-701 (2002).

34. Bates, G.P. \& Gonitel, R. Mouse models of triplet repeat diseases. Mol Biotechnol 32, 147-158 (2006).

35. Ling, S.C., Polymenidou, M. \& Cleveland, D.W. Converging mechanisms in ALS and FTD: disrupted RNA and protein homeostasis. Neuron 79, 416-438 (2013).

36. Thomas, M., Alegre-Abarrategui, J. \& Wade-Martins, R. RNA dysfunction and aggrephagy at the centre of an amyotrophic lateral sclerosis/frontotemporal dementia disease continuum. Brain 136, 1345-1360 (2013).

37. Sobczak, K., de Mezer, M., Michlewski, G., Krol, J. \& Krzyzosiak, W.J. RNA structure of trinucleotide repeats associated with human neurological diseases. Nucleic acids research 31, 5469-5482 (2003).

38. Kuroyanagi, H. Fox-1 family of RNA-binding proteins. Cell Mol Life Sci 66, 3895-3907 (2009).

39. Ying, Y., et al. Splicing Activation by Rbfox Requires Self-Aggregation through Its Tyrosine-Rich Domain. Cell 170, 312-323 e310 (2017).

40. Schneider, C.A., Rasband, W.S. \& Eliceiri, K.W. NIH Image to ImageJ: 25 years of image analysis. Nat Methods 9, 671-675 (2012).

41. Shen, S., et al. MATS: a Bayesian framework for flexible detection of differential alternative splicing from RNA-Seq data. Nucleic acids research 40, e61 (2012). 


\section{FIGURE LEGENDS}

\section{Figure 1. Workflow of the technical approach.}

Steps for human/mouse intersect-RNA-seq analysis of neurodegenerative disease-specific missplicing and identification of underlying altered splicing factors and pathogenic effectors.

Figure 2. Intersect-RNA-seq analysis of striatum of HD patients and early symptomatic R6/1 mice.

(a) For at par RNA-seq analysis, polyA+ RNA was prepared from post-mortem striatum of HD patients (Vonsattel's grade 3-4) and matching control subjects ( $n=3)$, and from striatum of 3.5 month-old R6/1 mice, together with matching controls $(n=3)$. The schematic drawings on the left depict coronal sections of human control and HD patient brain showing striatal atrophy and micrographs on the right show representative images of DARPP32 immunostaining of nonatrophied striatum in coronal sections of 3.5 month-old R6/1 mice as compared to WT mice.

(b) Analysis of levels of cell type-specific transcripts in human (left, $n=3$ ) and mouse (right, $n=3$ ) striatal RNA-seq samples using geometric mean of the CRPKM values of cell type marker genes of neurons, astrocytes, microglia and oligodendrocytes (Student's t-test; ${ }^{*} P<0.05, * * P<0.01$, $* * * P<0.001)$.

(c) Splicing was analyzed with vast-tools, rMATs and SANJUAN software and the Venn diagram shows the number of mis-spliced genes in $\operatorname{HD}$ patients $(5,747)$ and R6/1 mice $(2,071)$ with respect to controls, as well as the intersect of 949 mis-spliced genes common to both species ( $p$ $=6.1 \times 10-3$, two-sided Fisher's Exact test using only one-to-one orthologs with sufficient read coverage in both species as background; $\mathrm{N}=12882$ ).

(d) Ingenuity Pathways Analysis (IPA) of the 342 human/mice shared mis-spliced genes detected by single-program RNA-seq analysis shown in Supplementary Table 10. 


\section{Figure 3. Mis-splicing of neurodegeneration-causing genes.}

(a) The OMIM database was used to select, among the genes that are mis-spliced in both human and mouse striatum, genes whose mutations in humans cause monogenic forms of neurodegeneration.

(b) Using VastDB ${ }^{30}$, the orthologous alternative spliced events differentially processed in both species were identified and the PSI values of the mis-spliced events in each gene are shown in 3 WT vs 3 R6/1 samples (according to the RNA-seq vast-tools analysis).

(c) RT-PCR assay and quantification of mis-spliced events in striatal RNA from an independent set of WT and R6/1 mice $(n=5)$. (Student's t-test; $\left.{ }^{*} P<0.05,{ }^{* *} P<0.01\right)$. Data represent mean \pm SEM.

Figure 4. Decreased protein levels of neurodegeneration-causing genes in striatum of R6/1 mice.

Protein levels in striatum of WT and R6/1 mice and quantifications normalized with Vinculin ( $n=7-12)$. (Student's t-test; $\left.{ }^{*} P<0.05,{ }^{* *} P<0.01,{ }^{* * *} P<0.001\right)$. Data represent mean \pm SEM.

Figure 5. Human-mouse parallel RBP motif scan analysis in mis-regulated alternatively spliced events identifies enrichment of several splicing factor binding motifs.

(a) Schematic representation of scan RBP motif analysis performed in the 73 differentially included alternative exons detected with vast-tools and conserved in human (h) and mice (m). (b) Significantly enriched binding sites of splicing factors in 250 intronic base pairs adjacent to differentially skipped exons in HD and R6/1 samples.

Figure 6. Decreased protein levels of splicing factors in striatum of HD mouse model.

Protein levels in striatum of WT and R6/1 mice and quantifications normalized with $\beta$-actin ( $n=7$ -

12). (Student's t-test; ${ }^{*} P<0.05,{ }^{* *} P<0.01,{ }^{* * *} P<0.001$ ). Data represent mean \pm SEM. 
bioRxiv preprint doi: https://doi org/10.1101/2020.05.11.086017; this version posted May 12, 2020. The copyright holder for this preprint

(which was not certified by peer review) is the author/funder, who has granted bioRxiv a license to display the preprint in perpetuity. It is made available under aCC-BY-NC-ND 4.0 International license.

Figure 7. Alteration of splicing factors and neurodegeneration-causing genes protein levels in striatum of HD patients.

(a) Representative western blots of protein levels of splicing factors in striatum of HD patients and controls and quantification normalized with Vinculin ( $n=7-13)$. (Student's t-test; ${ }^{*} P<0.05$, ** $P<0.01, * * * P<0.001)$. Data represent mean \pm SEM.

(b) Localization of binding motifs of splicing factors enriched in the proximity of the mis-spliced events in each pathogenic gene and their protein levels in control and HD striatum. Histograms show quantification of protein levels normalized with Vinculin $(n=7-13)$. (Student's t-test; ${ }^{*} P<$ $0.05, * * P<0.01, * * * P<0.001)$. Data represent mean \pm SEM. 


\section{SUPPLEMENTARY FIGURE AND TABLE LEGENDS}

\section{Supplementary Fig. 1}

(a) Rotarod test. Mean latency to fall from the rod in the four accelerating testing trials of WT $(n=9)$ and R6/1 ( $n=11)$ mice at 3.5 months of age. (Student's t-test; $\left.{ }^{* *} P<0.01\right)$. Data represent mean \pm SEM.

(b) Representative image of toluidine blue staining in coronal sections from R6/1 mice and quantification of the number of neurons and the striatal volume measured in coronal sections of WT $(n=3)$ and R6/1 ( $n=3)$ mice at 3.5 months of age. (Student's t-test). Data represent mean \pm SEM.

(c) Representative images of Iba1 immunostaining in sagittal sections from R6/1 ( $n=3)$ and WT $(n=3)$ mice at 3.5 months of age and quantification of the density of microglial cells. (Student's t- test). Data represent mean \pm SEM.

\section{Supplementary Fig. 2}

Venn diagrams showing the number of mis-spliced genes in striatum of HD patients and R6/1 mice with respect to controls and those shared by both species, according to each of the ASanalysis tools.

\section{Supplementary Fig. 3}

Elavl3, Ptbp1 and Ptbp2 levels in WT and R6/1 striatum and quantification normalized with $\beta$ actin ( $n=7-12)$. (Student's t-test). Data represent mean \pm SEM. 
bjoRxiv preprint doi: https://doi.org/10.1101/2020.05.11.086017; this version posted May 12,2020 . The copyright holder for this preprint

(1) made available under aCC-BY-NC-ND 4.0 International license.

Human/mouse intersect-RNA-seq to capture neurodegenerative disease-specfic mis-splicing and identificatin of underlying altered splicing factors and pathogenic effectors

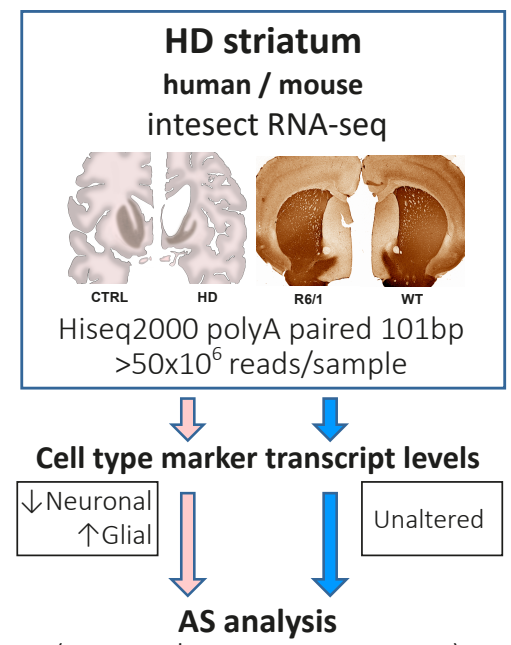

(vast-tools, rMATS, SANJUAN)

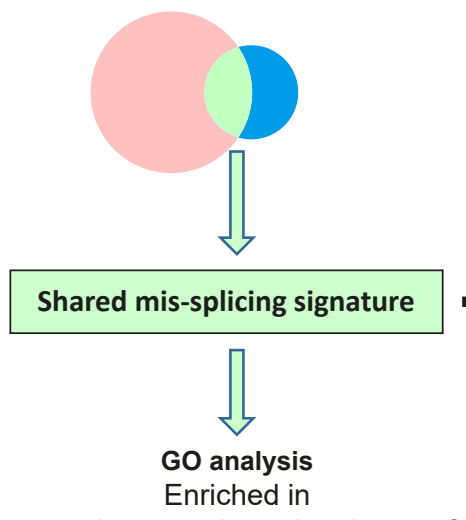

neurodegeneration-related genes?

\section{Huntington's disease}

\section{Pathogenic effectors}

RT-PCR validation and impact on protein levels in independent mouse model samples

Pathogenic effectors

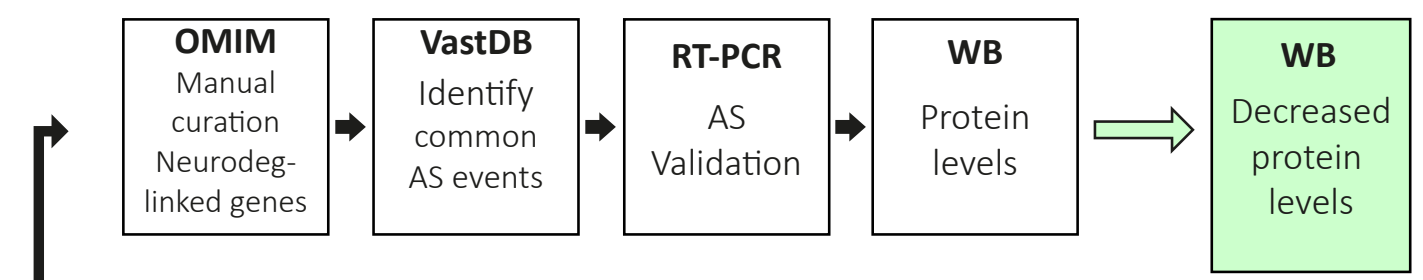

Human
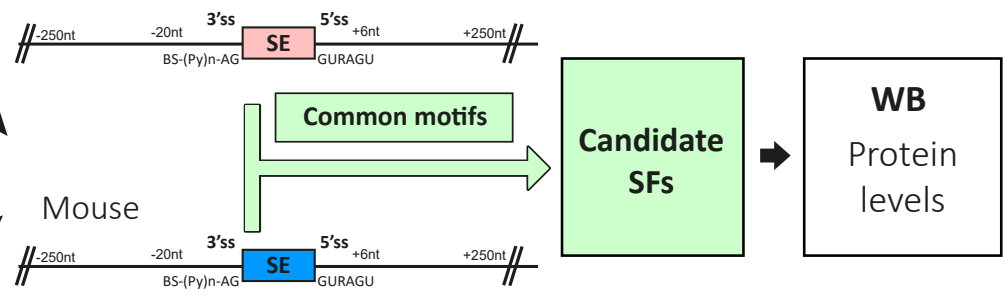

\section{Altered splicing factors}

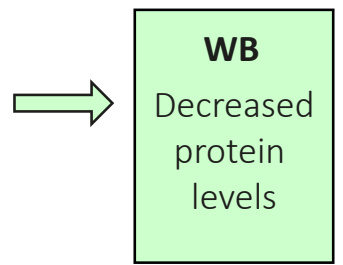

Parallel Motif Scan in mouse samples
Validate SF alteration

HD striatum

human

independent

samples

Validation<smiles>[C+]1=CC=1</smiles>

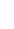

\begin{tabular}{|c|c|c|c|c|c|c|c|c|}
\hline $\begin{array}{c}\text { OMIM } \\
\text { Manual } \\
\text { curation } \\
\text { Neurodeg- } \\
\text { linked genes }\end{array}$ & $\Rightarrow$ & $\begin{array}{l}\text { VastDB } \\
\text { Identify } \\
\text { common } \\
\text { AS events }\end{array}$ & $\Rightarrow$ & $\begin{array}{c}\text { RT-PCR } \\
\text { AS } \\
\text { Validation }\end{array}$ & $\Rightarrow$ & $\begin{array}{c}\text { WB } \\
\text { Protein } \\
\text { levels }\end{array}$ & $\Longrightarrow$ & $\begin{array}{c}\text { WB } \\
\text { Decreased } \\
\text { protein } \\
\text { levels }\end{array}$ \\
\hline
\end{tabular}

ors

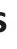 \\ Altered splicing factors}




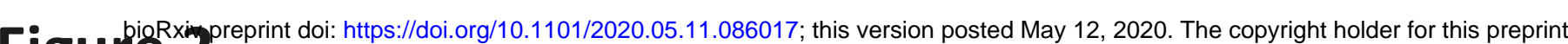

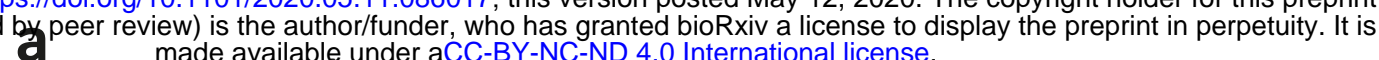
made available under aCG-BY-NC-ND 4 . International license.

\section{Striatum}

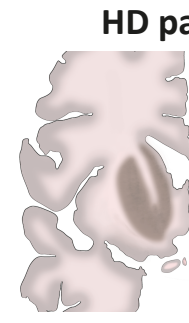

CTRL

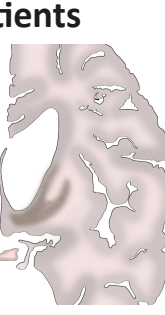

HD

$\mathbf{R} \mathbf{6 / 1}$ mice (early symptoms)

- Neuronal loss

- Gliosis

凡

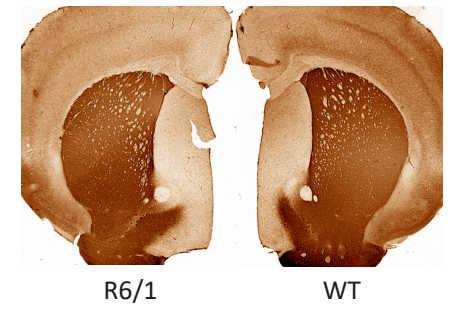

- No neuronal loss

- No gliosis

HiSeq2000, polyA+ paired 101bp $>50 \times 10^{6}$ reads/sample

b

\section{Cell type-specific transcript levels}

Neurons

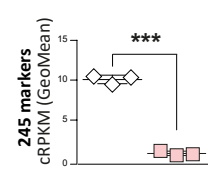

$\diamond \mathrm{CTRL}$

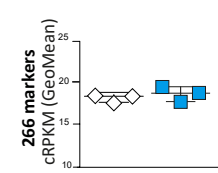

$\diamond W T$

Astrocytes
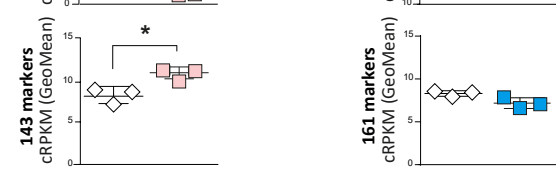

Microglia
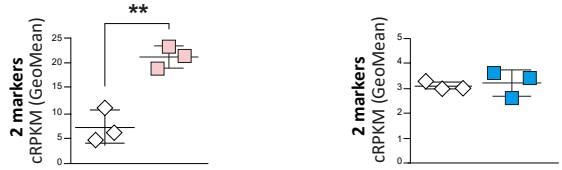

Oligo-
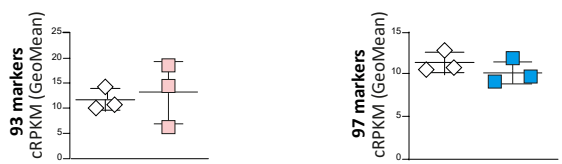

C

AS analysis

(vast-tools, rMATS, SANJUAN)

Mis-spliced

genes

HD

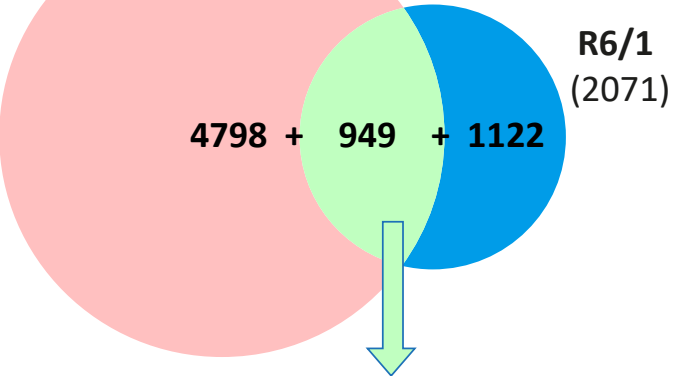

Shared mis-spliced genes

Movement disorder genes-enrichment

d

\begin{tabular}{|lcc|}
\hline Diseases or Functions Annotation & p-value & Gene number \\
\hline Movement Disorders & $\mathbf{2 , 3 0 E - 0 8}$ & $\mathbf{5 5}$ \\
Abnormal morphology of nervous system & $6,59 \mathrm{E}-08$ & 44 \\
Disorder of basal ganglia & $\mathbf{8 , 5 1 E - 0 8}$ & $\mathbf{4 1}$ \\
Familial encephalopathy & $6,70 \mathrm{E}-07$ & 54 \\
Abnormal morphology of neurons & $1,41 \mathrm{E}-06$ & 29 \\
Neuromuscular disease & $\mathbf{1 , 7 0 E}-06$ & $\mathbf{4 3}$ \\
Progressive encephalopathy & $2,25 \mathrm{E}-06$ & 41 \\
Progressive neurological disorder & $\mathbf{2 , 3 4 E - 0 6}$ & $\mathbf{4 6}$ \\
Degenerative dementia & $\mathbf{4 , 4 1 E - 0 6}$ & $\mathbf{3 1}$ \\
Dementia & $\mathbf{7 , 0 8 E - 0 6}$ & $\mathbf{3 2}$ \\
\hline
\end{tabular}


Fi

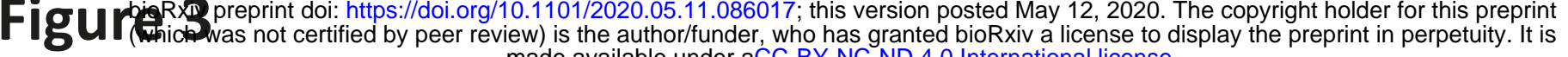
made available under aCC-BY-NC-ND 4.0 International license.

a

\section{OMIM}

Neurodeg.-

linked genes

Gene/

Disease

CCDC88C

SCA40

KCTD17

DYT26

SYNJ1

PARK20

VPS13C

PARK23

TRPM7

ALSP/DC

SLC9A5

EKD2* b

$$
\begin{gathered}
\text { VastDB } \\
\text { ortologous } \\
\text { events }
\end{gathered}
$$

mouse

RNA-seq data

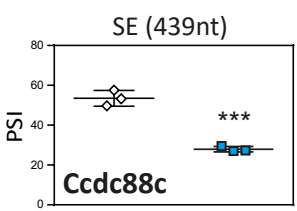

SE (75nt)
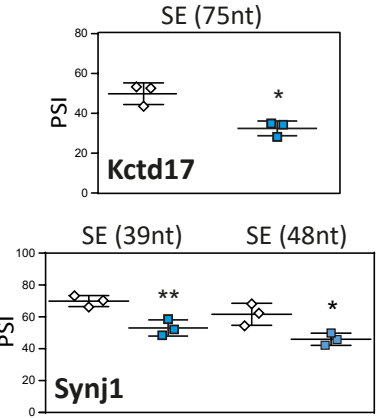

SE (63nt)
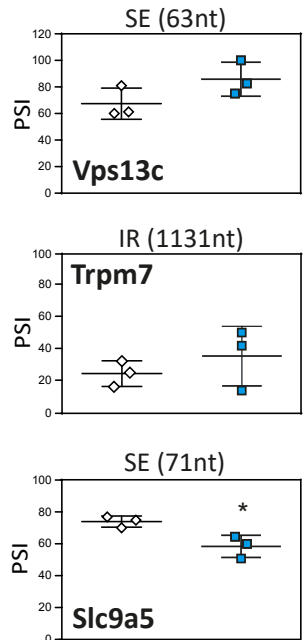

C

\section{RT-PCR}

Validation

independent mouse samples

$\diamond \mathrm{WT}$

$\square \mathrm{R} 6 / 1$
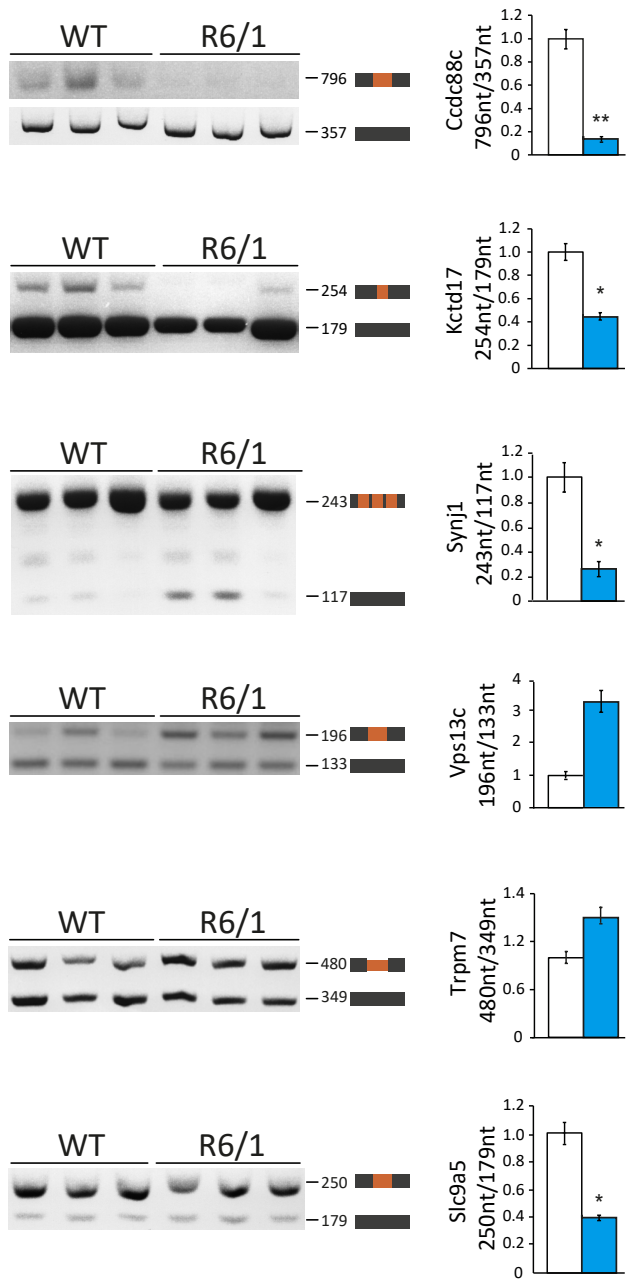

$\square$ WT

R6/1 
bioRxiv preprint doi: https://doi. org/10 1101/2020 05.11.086017· this version posted May 12,2020 . The copyright holder for this preprint made available under aCC-BY-NC-ND 4.0 International license.
mas not certified by peer review) is the authore the preprint in perpetuity. It is
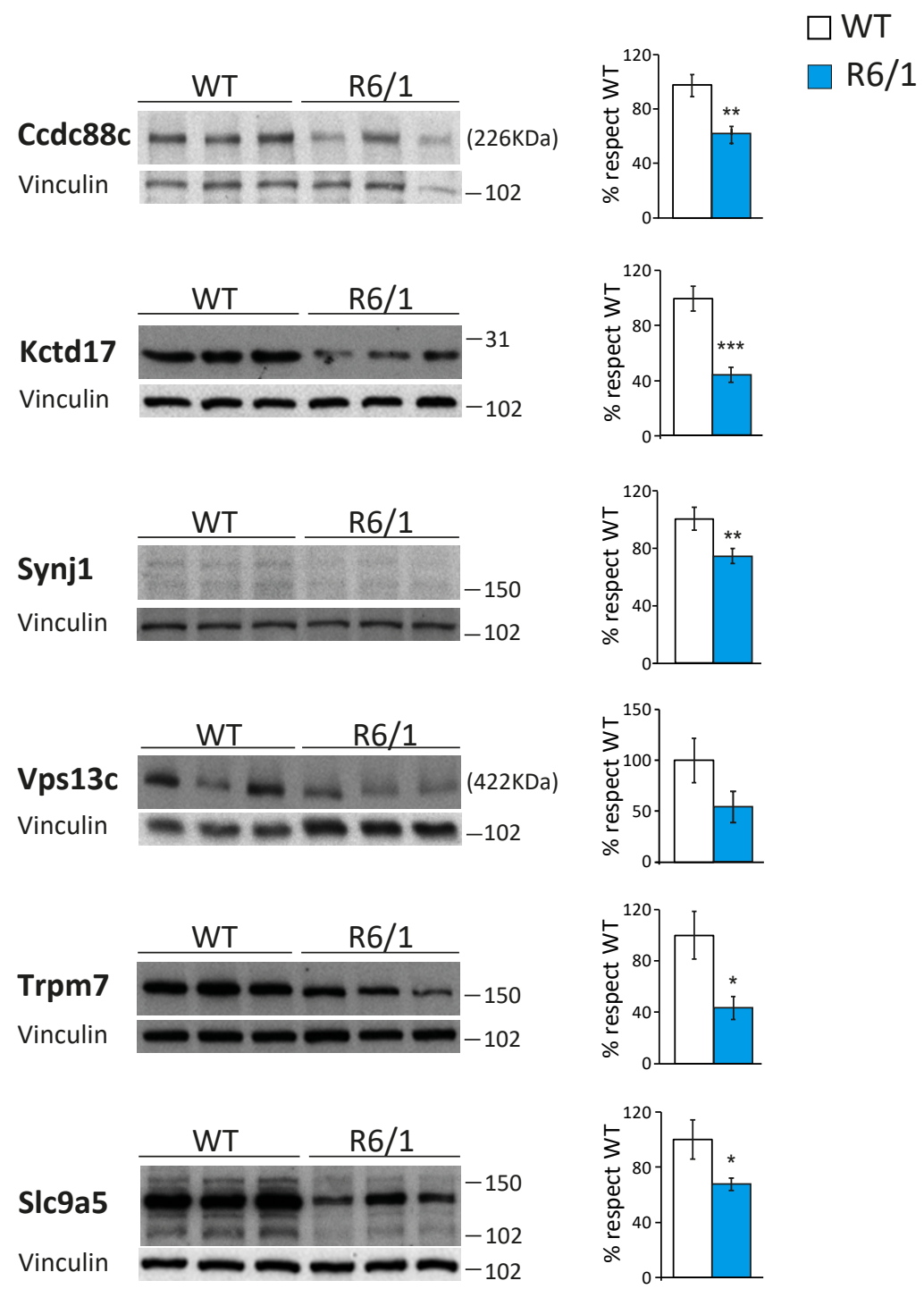


\section{Parallel Motif Scan}

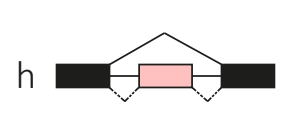

VS.

h

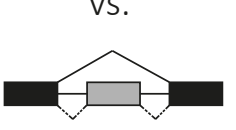

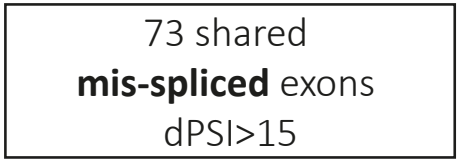

914 conserved

Control AS exons

$\mathrm{dPS} \mid<5$

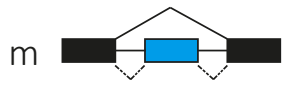

VS.

$\mathrm{m}$

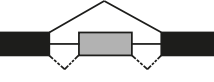

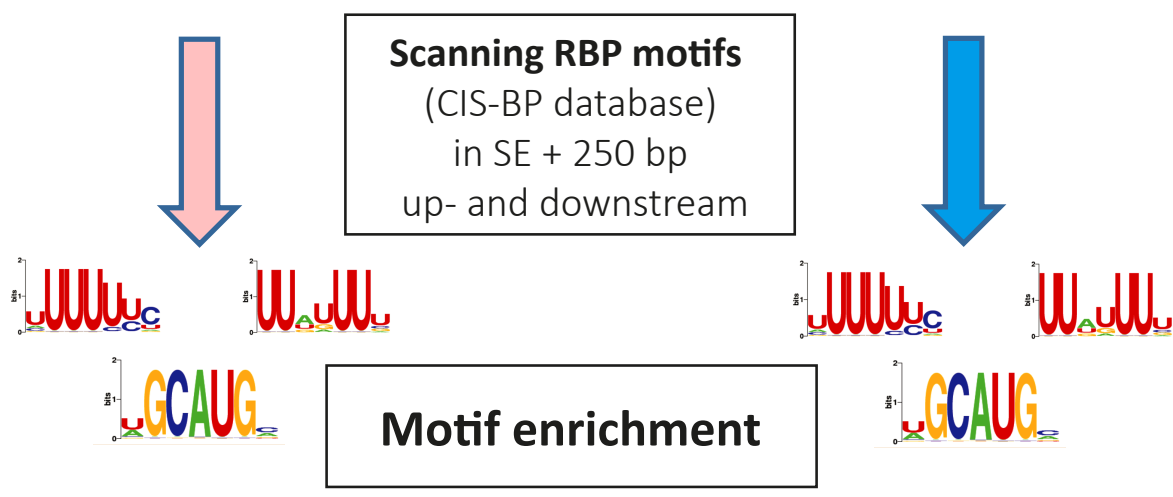

b

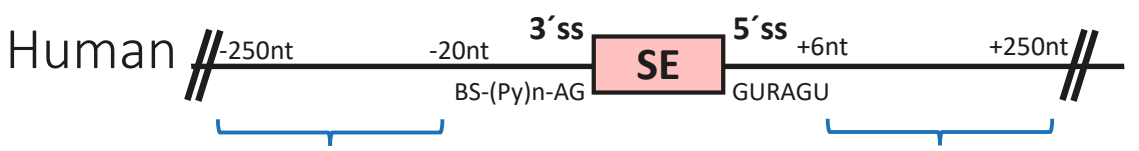

TIA1

Coincidental

U2AF2

RBFOX-1,-2,-3

RBP motifs

HNRNPC

ELAVL-1,-2,-3,-4

PTBP-1,-2

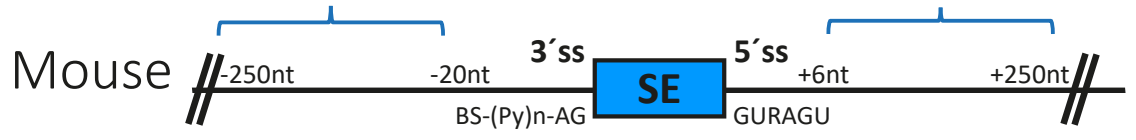

\begin{tabular}{|c|c|c|c|c|c|}
\hline \multirow{2}{*}{ Splicing Factor } & \multirow{2}{*}{ Binding motif } & \multicolumn{2}{|c|}{ Human } & \multicolumn{2}{|c|}{ Mice } \\
\hline & & p-val & adj p-val & p-val & adj p-val \\
\hline \multicolumn{6}{|l|}{ 3' SS } \\
\hline TIA1 & UUUUUBK & $1,607 \mathrm{E}-06$ & $1,141 \mathrm{E}-04$ & $2,002 \mathrm{E}-06$ & $1,261 \mathrm{E}-04$ \\
\hline U2AF2 & UUUUUYC & 2,657E-07 & 1,913E-05 & 5,070E-06 & 3,143E-04 \\
\hline HNRNPC & HUUUUUK & $1,713 \mathrm{E}-05$ & $1,182 \mathrm{E}-03$ & 3,516E-05 & $2,075 \mathrm{E}-03$ \\
\hline PTBP-1,-2,-3 & HYUUUYU & 1,139E-08 & 8,427E-07 & $2,009 \mathrm{E}-12$ & $1,306 \mathrm{E}-10$ \\
\hline \multicolumn{6}{|l|}{$5^{\prime} \mathrm{SS}$} \\
\hline RBFOX-1,-2,-3 & $\overline{\text { WGCAUG }}$ & $9,688 \mathrm{E}-07$ & $7,072 \mathrm{E}-05$ & $1,405 \mathrm{E}-04$ & $8,851 \mathrm{E}-03$ \\
\hline ELAVL-1,-2,-3,-4 & UUDUUUU & $8,126 \mathrm{E}-08$ & 6,094E-06 & $1,698 \mathrm{E}-06$ & 1,087E-04 \\
\hline
\end{tabular}



Figurigeri made available under aCC-BY-NC-ND 4.0 International license.
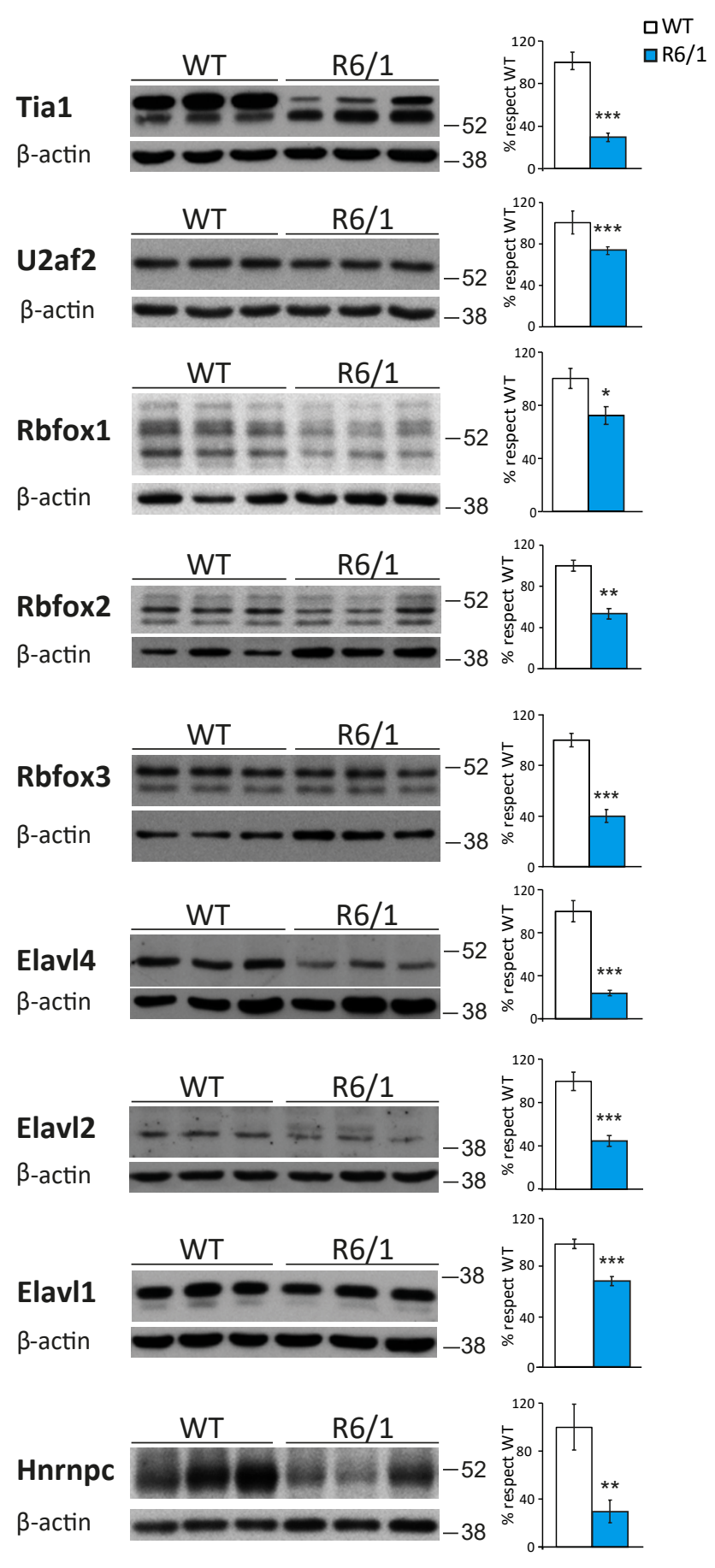
bioRxiv preprint doi: $\mathrm{https}$ //doi. org/10.1101/2020.05.11.086017 t this version posted May 12,2020 . The copyright holder for this preprint

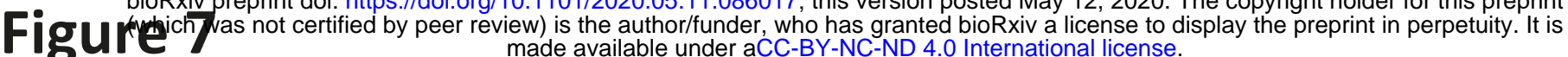
made available under aCC-BY-NC-ND 4.0 International license.

a

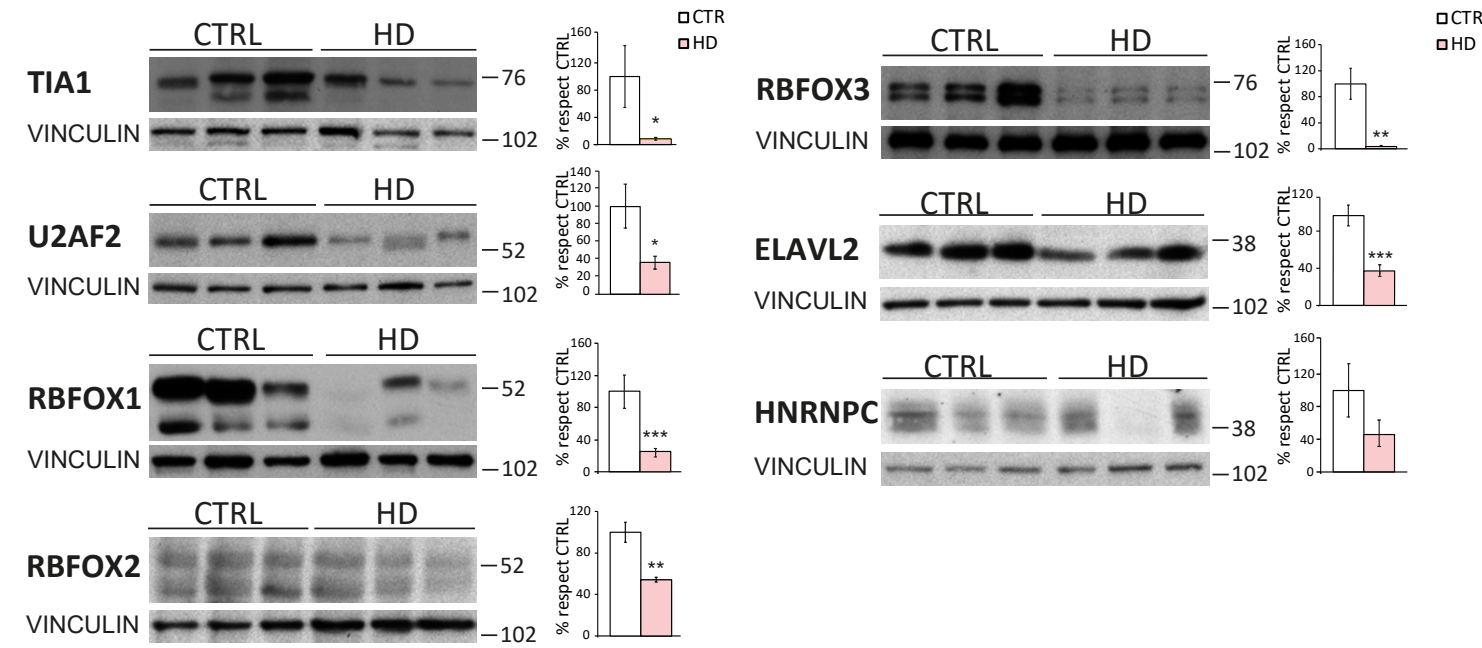

b

\section{Pathogenic target genes}

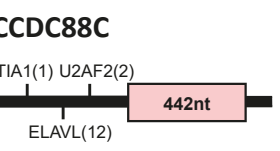

KCTD17
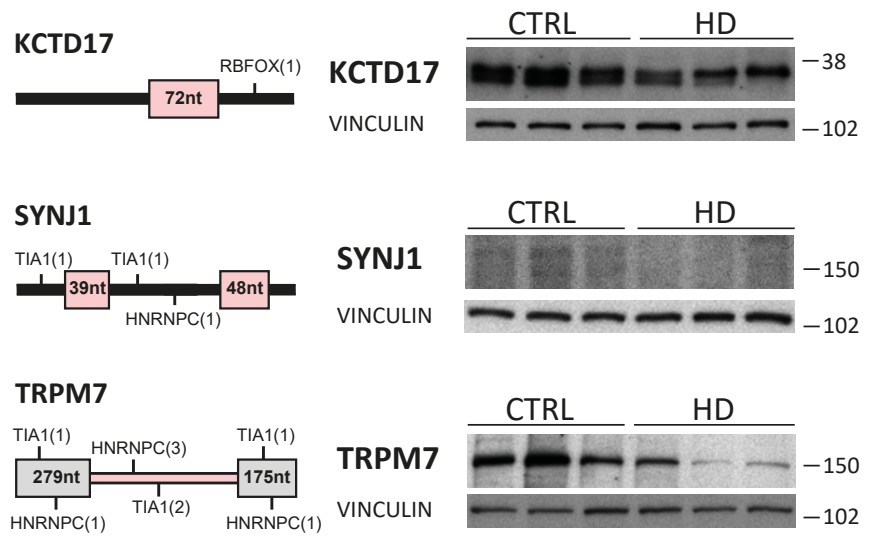

TRPM7
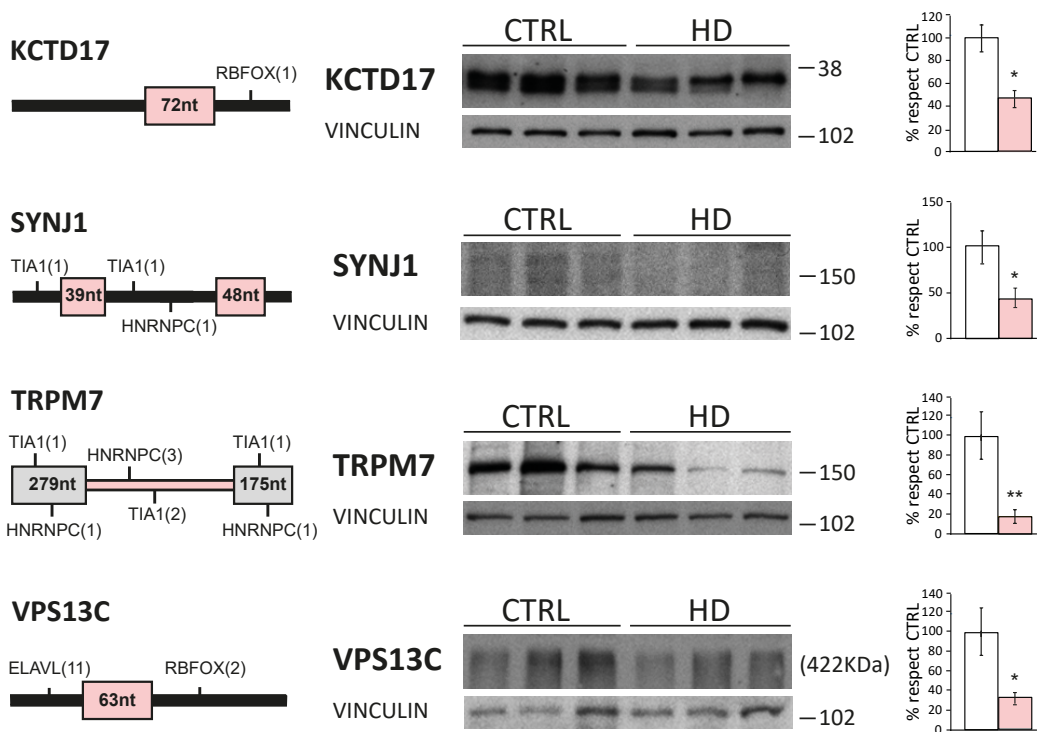

SLC9A5

ELAVL(1) RBFOX(4)

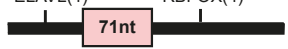

SLC9A5

VINCULIN

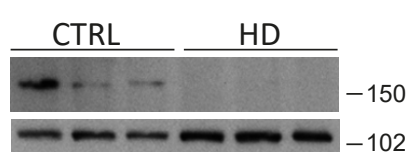

DYT27

PARK20

Associated

disease

SCA40

ALS/DC

PARK23

EKD2* 
a

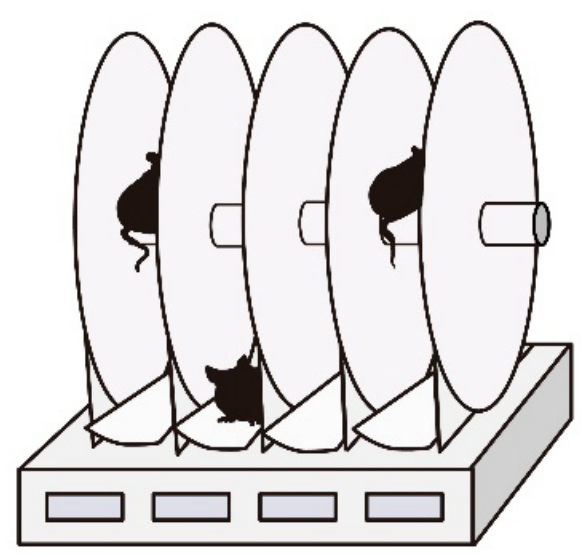

\section{Rotarod}

3.5 months

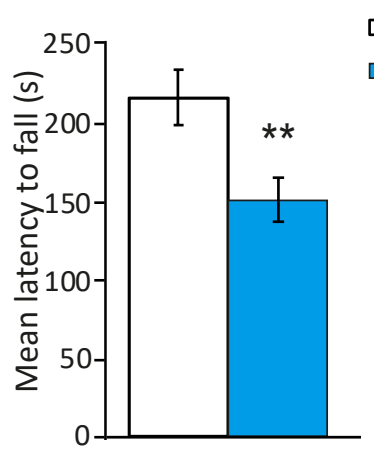

b

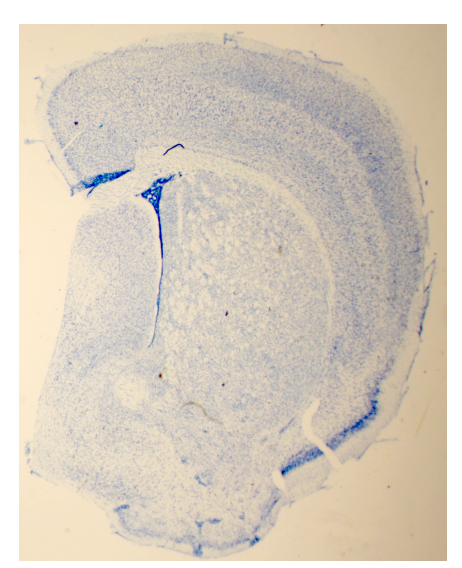

C

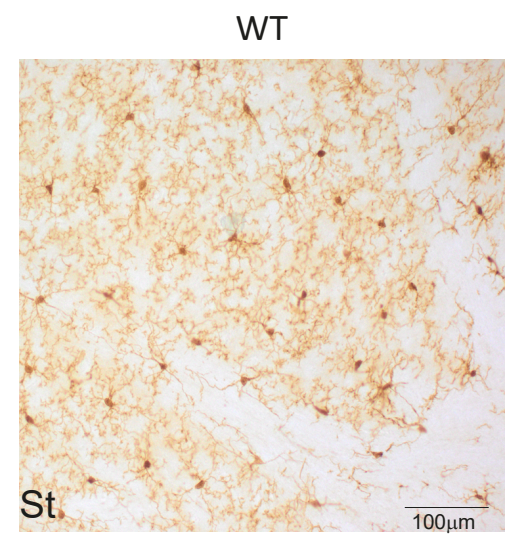

Striatal volume

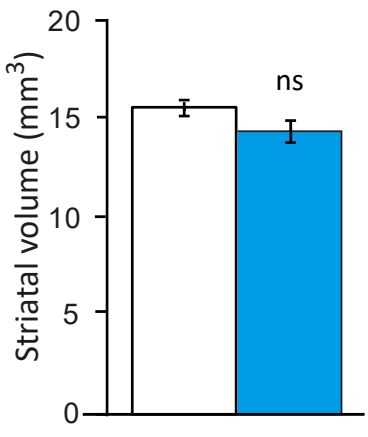

R6/1

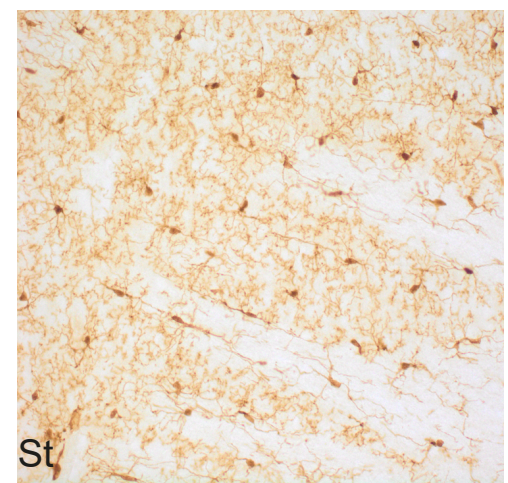

口WT

$\mathrm{R} 6 / 1$
Number of striatal neurons

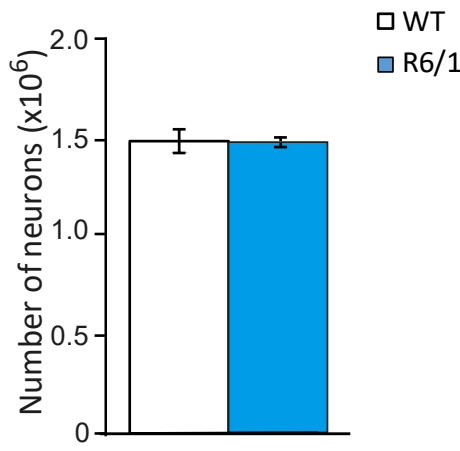

Microglial density

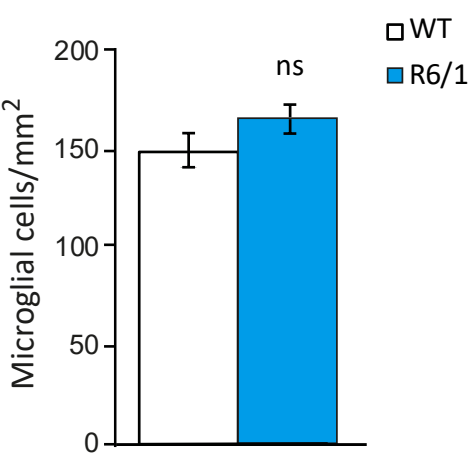


Single program-detected shared mis-spliced genes

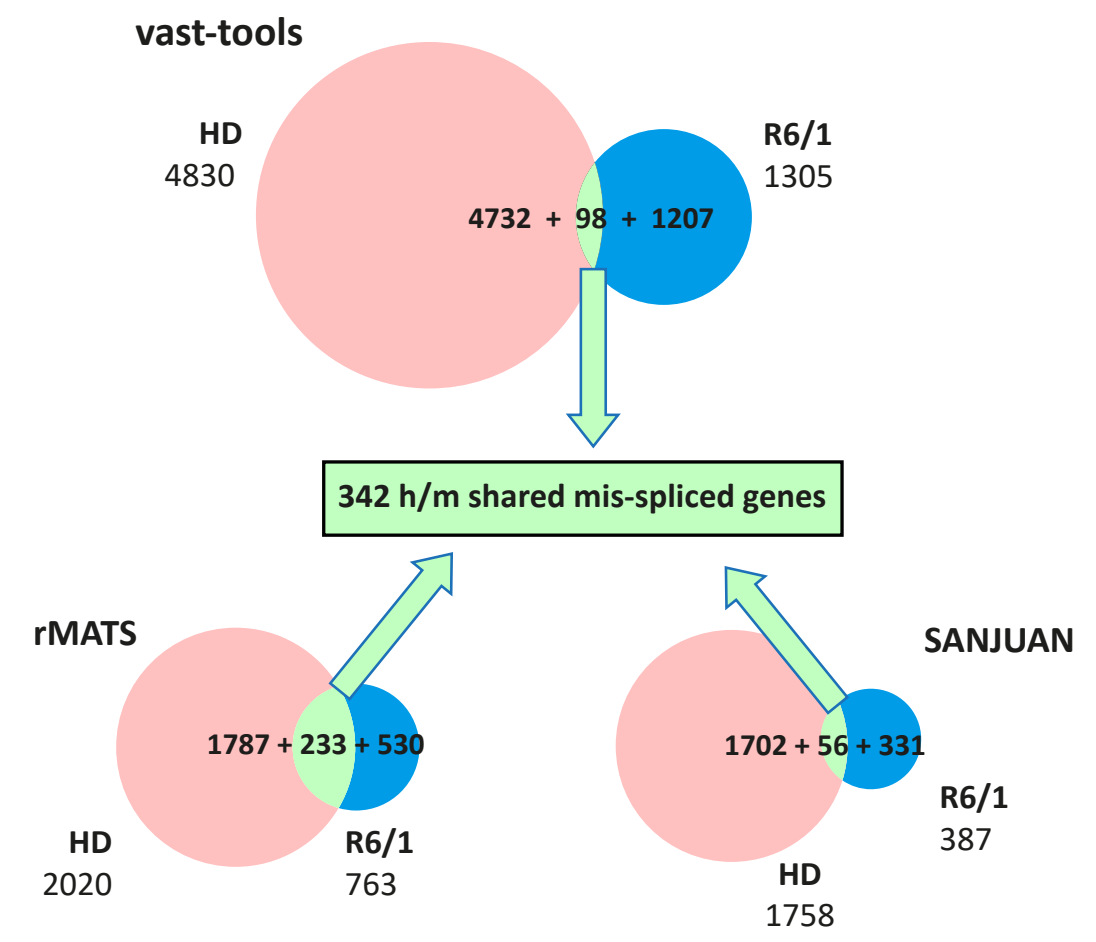




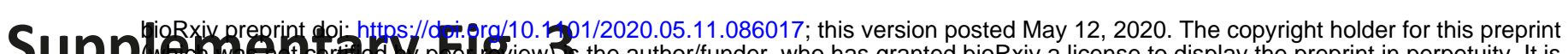
made available under aCC-BY-NC-ND 4.0 International license.
made the author/funder, who has granted bioRxiv a license to display the preprint in perpetuity. It is
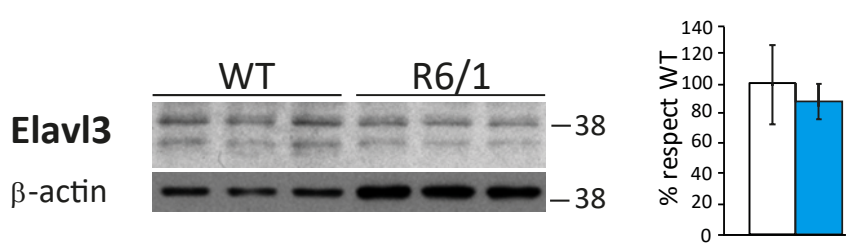

Ptbp1
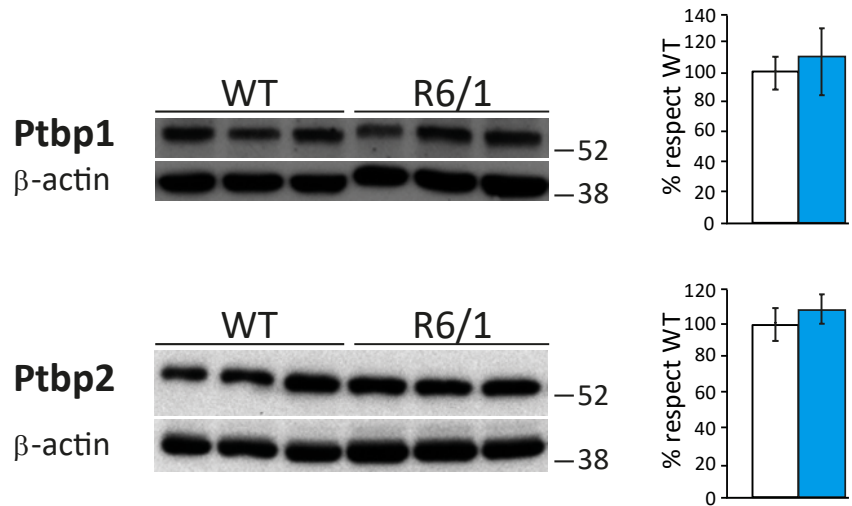